\title{
Switching of ferroelectric liquid crystal doped with cetyltrimethylammonium bromide-assisted CdS nanostructures
}

\author{
Kaushik Pal , Uday Narayan Maiti , Tapas Pal Majumder , \\ Subhas Chandra Debnath , Sharmistha Ghosh-, Subir Kumar Roy and \\ José Manuel Otón
}

\begin{abstract}
Large scale high yield cadmium sulfide (CdS) nanowires with uniform diameter were synthesized using a rapid and simple solvo-chemical and hydrothermal route assisted by the surfactant cetyltrimethylammonium bromide (CTAB). Unique CdS nanowires of different morphologies could be selectively produced by only varying the concentration of CTAB in the reaction system with cadmium acetate, sulfur powder and ethylenediamine. We obtained CdS nanowires with diameters of $64-65 \mathrm{~nm}$ and lengths of up to several micrometers. A comparative study of the optical properties of ferroelectric liquid crystal (FLC) Felix-017/100 doped with 1\% of CdS nanowires was performed. Response times of the order of from 160 to $180 \mu \mathrm{s}$, rotational viscosities of the order of from 5000 to $3000 \mathrm{mN} \mathrm{s} \mathrm{m}^{-2}$ and polarizations of the order of from 10 to $70 \mathrm{nC} \mathrm{cm}^{-2}$ were measured. We also observed an anti-ferroelectric to ferroelectric transition for CdS doped FLC instead of the ferroelectric to paraelectric transition for pure FLC.
\end{abstract}

(Some figures may appear in colour only in the online journal)

\section{Introduction}

Quasi-one-dimensional nanostructure materials, such as nanowires and/or nanotubes, have been successfully synthesized and have received much attention because of their interesting physical properties and potential applications in nanodevices [1]. In recent years, synthesis of one-dimensional semiconductor materials such as nanowires, nanorods and fibers has been the focus of a number of research works [2-5]. $\mathrm{CdS}$ is one of the most important II-VI semiconductors. It is a direct-band semiconductor with a band gap of $2.4 \mathrm{eV}$. CdS has vital optoelectronic applications for laser light emitting diodes and optical devices based on nonlinear properties [6]. As CdS is an important II-VI semiconductor material, CdS nanocrystals have received considerable interest from researchers regarding the control of its morphology and size. Various approaches, such as the solvothermal route [7-9], a liquid crystal template, [10] irradiation [11, 12], polymer controlled growth [13] and electrodeposition on a porous template [14] have been applied to achieve one-dimensional CdS nanocrystals. Unfortunately, most of the reported nanowires have been very short, usually tens to several hundred nanometers in width and also in length. However, as we show in this work, it is possible to create nanowires having uniform lengths and widths with high yield in a facile unique strategy by the assistance of cetyltrimethylammonium bromide (CTAB).

Once the nanowire synthesis is well established, the goal is to employ the nanowires as dopant to modify the electro-optical properties of ferroelectric liquid crystals 
(FLCs), with the aim of improving their response in order to enhance their behavior in display and optoelectronics devices.

It is essential to obtain stable and uniform alignment of liquid crystals (LCs) on a macroscopic scale for the fabrication of high-quality liquid crystal displays (LCDs). Typically, alignment involves the modification or conditioning of a solid substrate such that its interface with the LC has some anchoring action that results in either planar (tangential) or homeotropic (perpendicular) orientation of the LC director (symmetry axis) with respect to the interface. Such conditioning is carried out on a substrate having an electrically conductive layer (usually indium tin oxide (ITO) or ITO-coated glass) for electric-field-induced reorientation of the director which, in turn, results in a variation in the transmitted light intensity. Currently, the preferred conditioning technique is rather primitive. The conductive substrate is coated with a polyimide layer that is mechanically rubbed $[15,16]$ after thermal curing. The alignment mechanism associated with unidirectional rubbing has contributions from both the physical grooves caused by rubbing the polyimide substrate, with the cost of the elastic energy related to conformably wetting the irregular substrate topography [15] and, secondly, putative molecular interactions between exposed polyimide functionalities and the LC. However, the details of LC alignment are not well understood [15, 17-19]. Thus, the search for more easily quantified alignment materials continues. The phase sequence of FLC Felix-017/100 mixture (Clairant, Frankfurt, Germany) is given by the following [20-22].

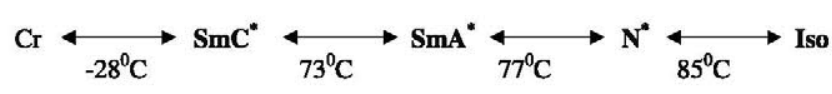

One-dimensional (1D) nanostructured materials doped with ferroelectric liquid crystals [23] are being intensively investigated because they impart remarkable improvement in the physical parameters of the host materials [24-32]. Novel functionalities are elicited depending upon the optical and electrical properties of the nanomaterials and the interaction of the nanomaterials with the orientational field of the liquid crystals. The Freedericksz threshold voltage, residual DC, driving voltages, etc are found to decrease in carbon nanotube or semiconducting nanorod driven nematic liquid crystals [27-29, 31]. Metal nanoparticles protected with ligand molecules introduced into a nematic liquid crystal host exhibit a frequency modulation response with a short response time of the order of milliseconds in twisted nematic geometry $[32,33]$. Doping ferroelectric nanoparticles in nematic LC increases the order parameter and phase sequence temperature range of the LC due to the effective coupling of the spontaneous polarization of the nanoparticles with the LC director [34-36]. Recently, a few works on doping nanoparticles in ferroelectric liquid crystals (FLCs) have been carried out [37-39]. Since the discovery of surface stabilization of ferroelectric liquid crystals [39], they have been considered as good candidates for display applications owing to their fast response and bistability. However, commercial applications in displays demand further improvements of the physical properties of the FLCs to yield better contrast, lower driving voltage and good alignment without chevron structures. Therefore, modification of the existing FLC by blending nanomaterials in it may be a prospective method to improve those properties. The use of semiconducting nanowires can improve the electro-optical properties of a ferroelectric liquid crystal. The improvements are significant in response time and rotational viscosity.

This study is directed at understanding the structure and physical properties of hydrogen bonded LC gels in freely suspended film geometry. The experiments include structural assessment by means of polarizing microscopy, electrooptical investigations and some accompanying spectroscopic measurements. We will discuss the influences of the network formed in the gelling process on the mesophase properties of the material, i.e. the stabilization of orientational states and the dynamics of the reorientation in electric fields, and on the mechanical properties such as shape distortions and the suppression of macroscopic flow in the films.

\section{Experimental procedure}

\subsection{Synthesis}

All the chemicals used in this work were of analytical grade and commercially available, and were used without further purification. The chemicals used, such as ethylenediamine (EDA), $\mathrm{Cd}\left(\mathrm{CH}_{3} \mathrm{COO}\right)_{2} \cdot 2 \mathrm{H}_{2} \mathrm{O}$, sulfur powder and CTAB, were purchased from Atlanta Drugs Chemical Reagent Co. Ltd (Sigma Aldrich and E Merck).

In a typical procedure, $0.66 \mathrm{~g}$ of $\mathrm{Cd}(\mathrm{Ac})_{2}$ and $0.075 \mathrm{~g}$ of sulfur $(S)$ powder were placed in a $100 \mathrm{ml}$ beaker, and were dissolved into $45 \%$ of ethylenediamine under vigorous stirring for $15 \mathrm{~min}$. Then, $0.35 \mathrm{~g}$ of CTAB was added to the precursor and then stirred for $25 \mathrm{~min}$. After a complete stirring using a magnetic stirrer (Remi), the final solution was placed in a $100 \mathrm{ml}$ stainless steel coated Teflon-lined autoclave. The autoclave was placed in a heating chamber at $200^{\circ} \mathrm{C}$ for 2 h. Finally, after cooling the autoclave naturally at room temperature, a strongly pungent bubble forming solution was produced. The resultant sample was filtered off and washed with deionized distilled water and ethanol several times until the impurity product was washed and separated away from the desired sample. The sample was dried out in a vacuum at $75^{\circ} \mathrm{C}$ for $3 \mathrm{~h}$. In order to study the influence of CTAB surfactant on the nanostructure formation, the original $0.35 \mathrm{~g}$ of CTAB was changed by repeating the same synthesis with 0.15 and $0.65 \mathrm{~g}$ of CTAB in a similar fashion.

Our main purpose in this paper is to investigate how the electro-optical properties change with the diameter and length of CdS nanowires at different ambient conditions of time. The test cells for dynamic measurements were prepared using $0.7 \mathrm{~mm}$ ITO-coated polished glass plates having $16(4 \mathrm{~mm} \times 4 \mathrm{~mm})$ pixels imprinted on the ITO coating by photolithography. Homogeneous planar alignment was induced on the ITO-coated glass plates which were spin-coated with CdS nanostructure samples. The thickness of the cells was about $0.1 \mu \mathrm{m}$, thin enough to allow surface stabilization of the specimen. Slices mounted on the glass 


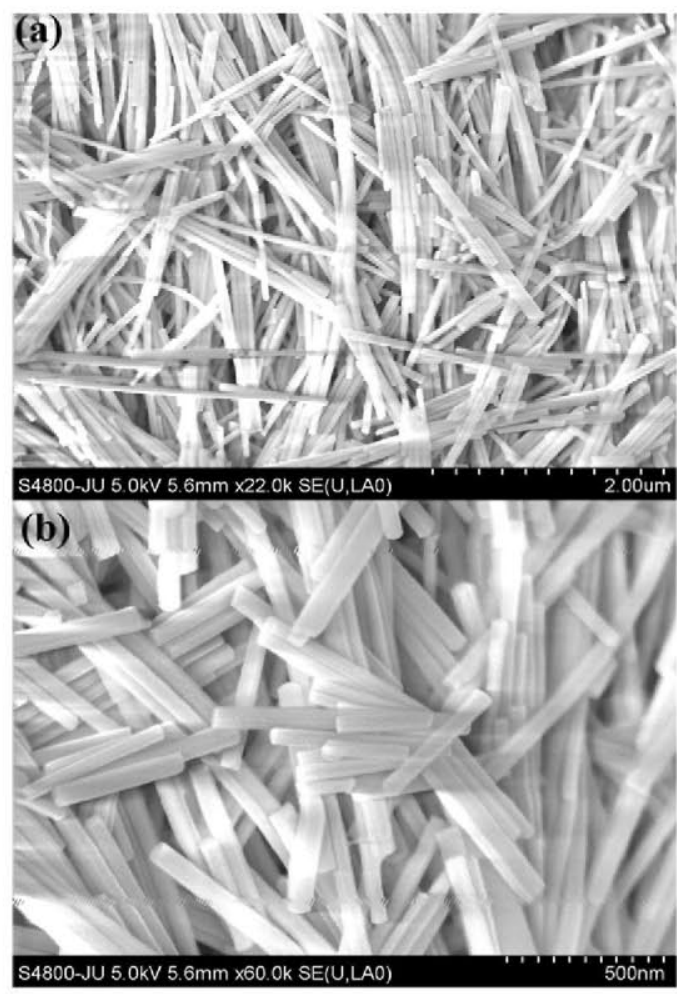

Figure 1. FE-SEM images of high yield uniform CdS nanowires evolved with the addition of CTAB $(0.35 \mathrm{~g})$ at a resolution of (a) $2 \mu \mathrm{m}$ and (b) $500 \mathrm{~nm}$.

sheet on opposite sides of the hole served as electrodes. They provided the driving electric fields in the film plane. We studied the images of the films with a reflection microscope. All texture pattern images (maximum frame rate 25 frames $\mathrm{s}^{-1}$ ) were recorded with a Nikon Coolpix $990 \mathrm{CCD}$ camera. They were subsequently contrast amplified and processed digitally. The films were observed with a crossed linear polarizer. However, since a number of optical elements are in the light path (e.g. a prism that splits the incident and reflected beams), the polarization of the incident light is not perfectly linear but slightly elliptical. This has to be considered in the discussion of optical image analysis through nano-doped liquid crystal phase changes with temperature.

\subsection{Characterizations}

Field emission scanning electron microscopy (FE-SEM) (model: Hitachi-FESEM, S4800-JU at $5.0 \mathrm{kV}$ and SEM, JUMET) was used to record the scanning electron micrograph for the morphological analysis of the nanostructures. The structures of the nanomaterials were investigated by using $\mathrm{x}$-ray diffraction studies (XRD) with a diffractometer (model: Bruker D8 Advance) with the $\mathrm{Cu} \mathrm{K} \alpha$ line. Preparation of the thin films were carried out by using a programmable spin coater (Apex Instruments Co., model: SCU-2008C) and the surface behavior with roughness of the thin films prepared in this way was studied by using atomic force microscopy (AFM) (model: Veeco, diInnova). An

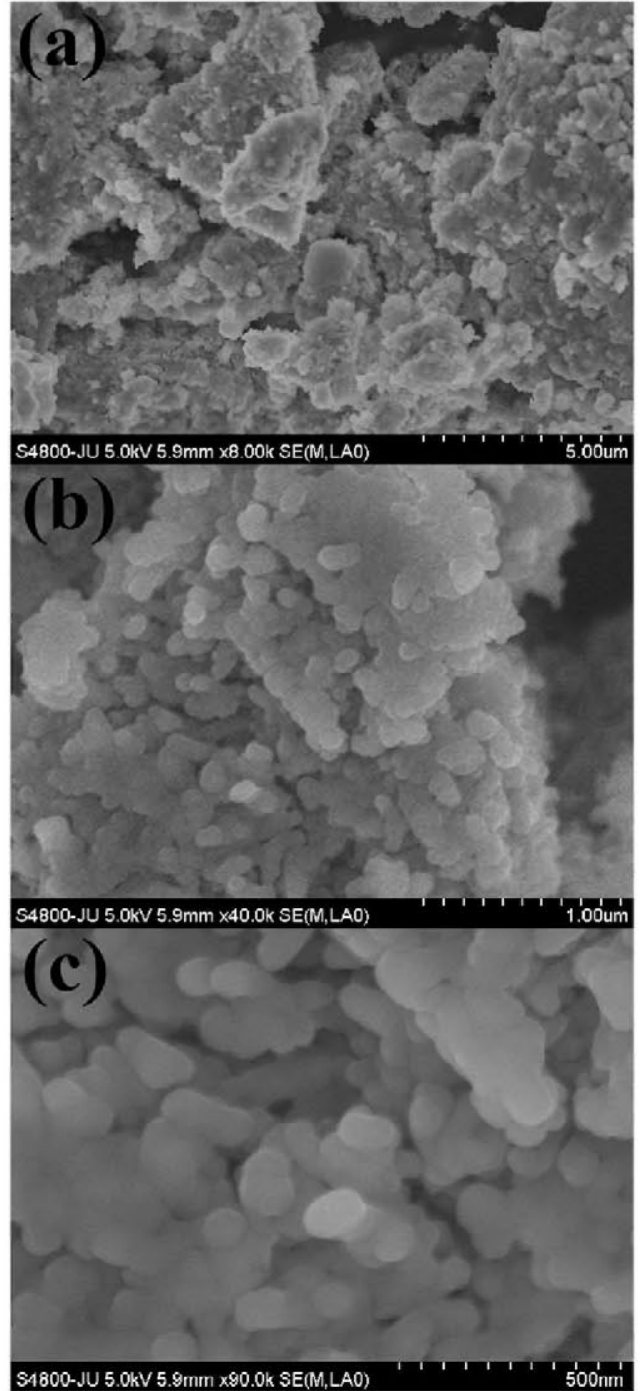

Figure 2. FE-SEM images of CdS nanoparticles evolved with the addition of CTAB $(0.15 \mathrm{~g})$ at a resolution of (a) $5 \mu \mathrm{m}$, (b) $1 \mu \mathrm{m}$ and (c) $500 \mathrm{~nm}$.

ultraviolet-visible scanning spectrophotometer (UV-2401PC, TCC-240A, Shimadzu, Japan) was used to record the electronic absorption spectra of the films at room temperature. The photoluminescence spectra were measured by using excitation of $325 \mathrm{~nm}$ from a $\mathrm{He}-\mathrm{Cd}$ laser source (LS55, fluorescence spectrometer, PerkinElmer). The frequency dispersion electro-optical data were recorded at room temperature for frequencies ranging from $10 \mathrm{~Hz}$ to $13 \mathrm{MHz}$ using a Hewlett Packard 4192A LF impedance analyzer and dc conductance measurements were made using a source meter (model: Keithley 2611A system).

For electro-optic measurement an HP 33120A signal generator and an HP 54603B oscilloscope were used. The spontaneous polarization $\left(P_{\mathrm{S}}\right)$ and the relaxation time were measured by using a polarization reversal method and calculated with the help of the following relation [30, 31]:

$$
P_{\mathrm{S}}=(1 / 2 A) \int i(t) \mathrm{d} t .
$$




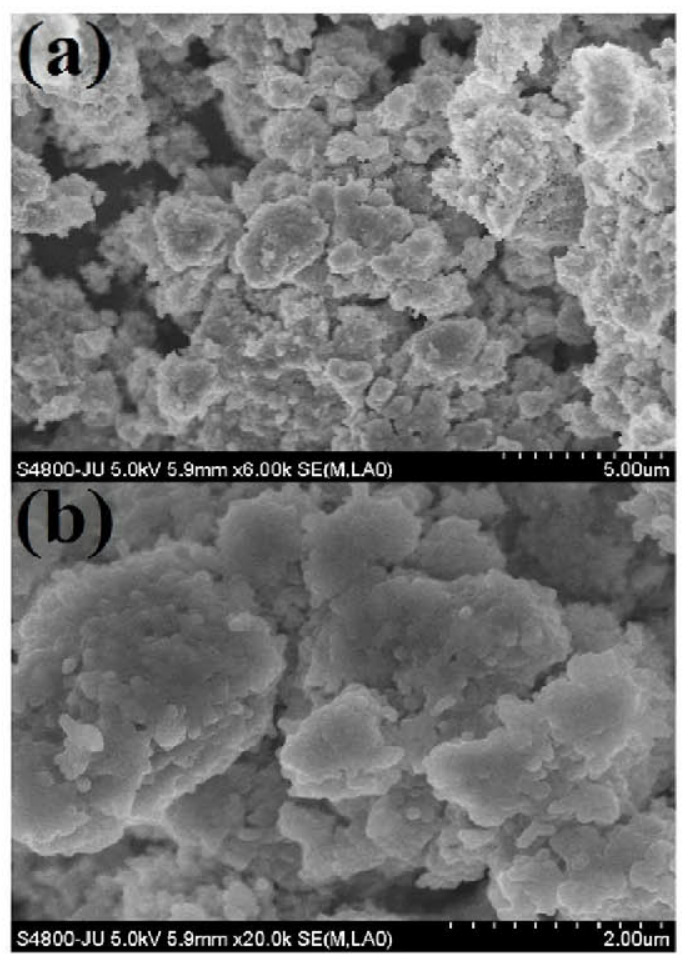

Figure 3. FE-SEM images of CdS nanogranules evolved with the addition of CTAB (0.65 g) at a resolution of (a) $5 \mu \mathrm{m}$ and (b) $2 \mu \mathrm{m}$.

All measurements were computer based. The viscosity $(\gamma)$ was determined from the measured value of the polarization $\left(P_{\mathrm{S}}\right)$ and the switching time $(\tau)$ using the following relation $[30,31]$ :

$$
\tau=\frac{\gamma}{1.75 P_{\mathrm{S}} E} .
$$

\section{Results and discussion}

\subsection{Morphology analysis}

The morphological confirmation of the different compositions of the CdS nanocomposite materials was performed using field emission scanning electron microscopy (FE-SEM). The experimental studies revealed that high yield large scale uniform CdS semiconductor nanowires with very uniform controllable morphological growth are synthesized using a unique solvothermal route with the addition of surfactant $\mathrm{CTAB}$, as shown in figure 1 . The results are significantly improved by using $\mathrm{CTAB}$ in comparison with previously reported [40] CdS nanostructures based on the surfactant polyethylene glycol (PEG) with various concentrations at different times and ambient conditions. In our earlier publication [41], we prepared CdS nanowires based on PEG surfactant with diameters in a range of the order of $31-36 \mathrm{~nm}$ and lengths in a range of the order of 354-360 nm. In this paper we have obtained CdS nanowires based on CTAB surfactant $(0.35 \mathrm{~g})$ having diameters of the order of $64-65 \mathrm{~nm}$ with lengths of the order of $434-473 \mathrm{~nm}$. Interestingly enough, by adding a lower amount of CTAB $(0.15 \mathrm{~g})$, the

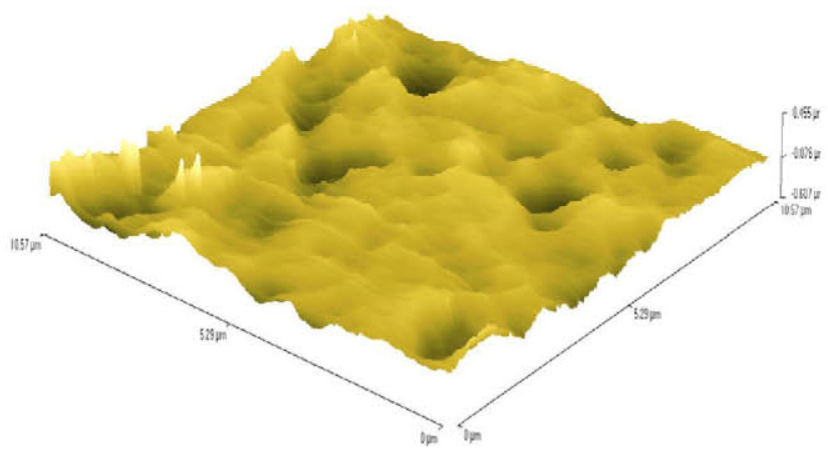

Figure 4. Atomic force microscopy (AFM) topography of as-prepared thin film for LC doped CdS nanowires $(0.35 \mathrm{~g} \mathrm{CTAB}$ concentration).

morphological structure of the CdS observed by SEM totally changed and the nanowires turned into nanoparticles with diameters of around 8-149 nm, as illustrated in figure 2. In addition to this, more interesting for this work is the result obtained at higher concentration $(0.65 \mathrm{~g})$. With the reaction of $\mathrm{CTAB}$ with ethylenediamine, conversion of $\mathrm{CdS}$ nanoparticles to nanogranule structures with a uniform diameter of $92 \mathrm{~nm}$ results, as confirmed in the higher resolution SEM imagee (figure 3(b)). This hydrothermal reaction takes place with ethylenediamine (en) acting as a solvent as well as a complexing agent. In our synthetic system, the investigation of CdS nanoparticle formation indicated that the nucleation and growth were well controlled. Firstly, ethylenediamine, as a strongly bidentating solvent, was ready to form relatively stable $\mathrm{Cd}^{2+}$ complexes [42-44]. Next, cadmium ions could combine with an ethylenediamine molecule (which acts as a bidentate ligand) to form a Cd-ethylenediamine complex $\left(\left[\mathrm{Cd}(\mathrm{en})_{2}\right]^{2+}\right)[42]$, which was stabilized in the solution. Meanwhile, $\mathrm{S}$ powder was dissolved in the ethylenediamine. Finally, due to the slow release of $\mathrm{S}^{2-}$ ions and the low free $\mathrm{Cd}^{2+}$ concentration $\left(\log \beta_{2}=10.09, \beta_{2}\right.$ is the stability constant of the complex $\left(\left[\mathrm{Cd}(\mathrm{en})_{2}\right]^{2+}\right)$ ), the reaction rate is slow, which is in favor of oriented growth of the CdS nanowires [45].

An atomic force microscope (AFM) is a kind of scanning probe microscope where imaging of the sample is realized by interaction of the probe with the sample surface and no imaging beam (light or electron) is involved in the process. This surface imaging technique involves scanning a sharp tip over the surface of a sample, while sensing the interaction force between the tip and the sample of a hybrid nanomaterial (LC doped CdS) specimen at sub-nanometer resolution under physiological conditions. In one form of AFM the force acting on the tip is measured with piconewton $(10-12 \mathrm{pN})$ sensitivity as the tip is pushed towards the sample and then retracted from it. Thin film sample preparations, recording conditions and surface behavior have revolutionized the way in which atomic force microscopy (AFM) can explore FLC (Felix) doped CdS nanomaterials. The thin films were spin-coated with spin speed adjustment of $500 \mathrm{rpm}$ in $20 \mathrm{~s}$, as depicted in figure 4. From figure 4 we can observe that the surface is uniformly distributed on a Pyrex cover slip (made in China) 

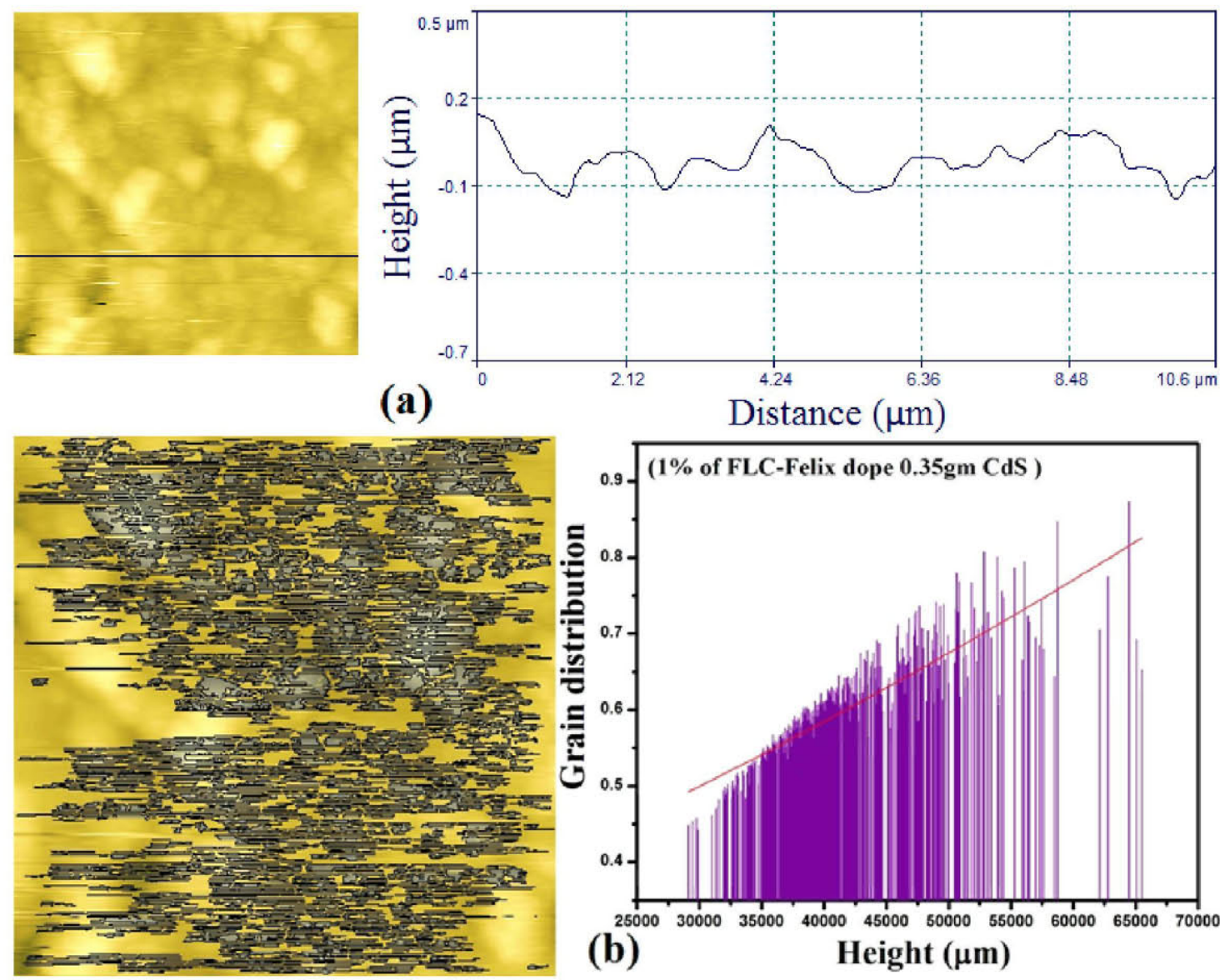

Figure 5. (a) A non-contact mode AFM image of LC doped CdS grain height profiles acquired at selected locations with distance. (b) The number density of grains plotted against height of the as-prepared thin film.

and the sample is homogeneously spread there. The average thickness of the thin film is $185 \mathrm{~nm}$. A detailed investigation is also made through further analysis of the AFM characteristics (figure 5(a)). In addition to this, we can also determine LC doped CdS nanomaterial thin films which vary with height distributed in nanometer ranges and we are able to predict the number density of the molecule (LC doped CdS grain) distribution; these increases are exponentially plotted against height in several micrometer ranges which resulted after analysis in figure 5(b). The thickness and roughness have been increased remarkably due to the introduction of Felix, keeping the same rpm for both cases for the preparation of a single layer homogeneous dispersion of thin film.

\subsection{Crystalline structure analysis}

We recorded x-ray diffraction patterns (XRDs) of pure CdS nanostructures, pure Felix and CdS doped Felix by using an $\mathrm{x}$-ray diffractometer with $\mathrm{Cu} \mathrm{K} \alpha$ radiation $(0.01418 \mathrm{~nm})$ at a scan rate of $0.05^{\circ} \mathrm{s}^{-1}$. Figure 6(b) shows the XRD pattern of the well crystalline synthesized CdS nanowires for the CTAB concentration of $0.35 \mathrm{~g}$, with all the diffraction peaks corresponding to the hexagonal wurtzite phase of CdS. From this XRD pattern we obtained the crystal parameters of the sample. These matched well with those on the JCPDS
Card (Joint Committee on Powder Diffraction Standards, Card No. 41-1049). For this XRD pattern the best crystalline peak occurred due to the (110) plane with the corresponding angle of $2 \theta \approx 45^{\circ}$. After changing the concentration of the surfactant CTAB to $0.15 \mathrm{~g}$, the crystalline structure shows a (002) plane with the corresponding angle of $2 \theta \approx 26.78^{\circ}$, as shown in figure $6(\mathrm{a})$. Going to a higher concentration of CTAB $(0.65 \mathrm{~g})$, three well crystalline peaks are also identified in the planes (110), (103) and (112) along with those observed with high intensity crystalline peak maxima in the (002) plane resulting due to nanogranule formation, as clearly shown in figure $6(\mathrm{c})$; these are also confirmed through FE-SEM morphology analysis. Again, the diffraction pattern of LC doped with an appropriate concentration of CTAB (0.35 g) demonstates appropriate and distinct well crystalline peaks originating due to the (100) and (110) planes with corresponding angles of $2 \theta \approx 25.04^{\circ}$ and $43.88^{\circ}$, respectively. This is due to the smooth specimen of uniform size nanowires homogeneously dispersed in liquid crystals, as shown in figure $6(\mathrm{~d})$. Only for pure Felix can no such prominent peak be detected, as shown in figure 6(e). The peaks in the figure are identified as originating from the (100), (002), (101), (110), (112) and (114) planes of the hexagonal wurtzite phase of CdS. No impurity peaks were detected, indicating a high purity product. In addition, the intense and sharp diffraction peaks for (101), (110) and (002) respectively 

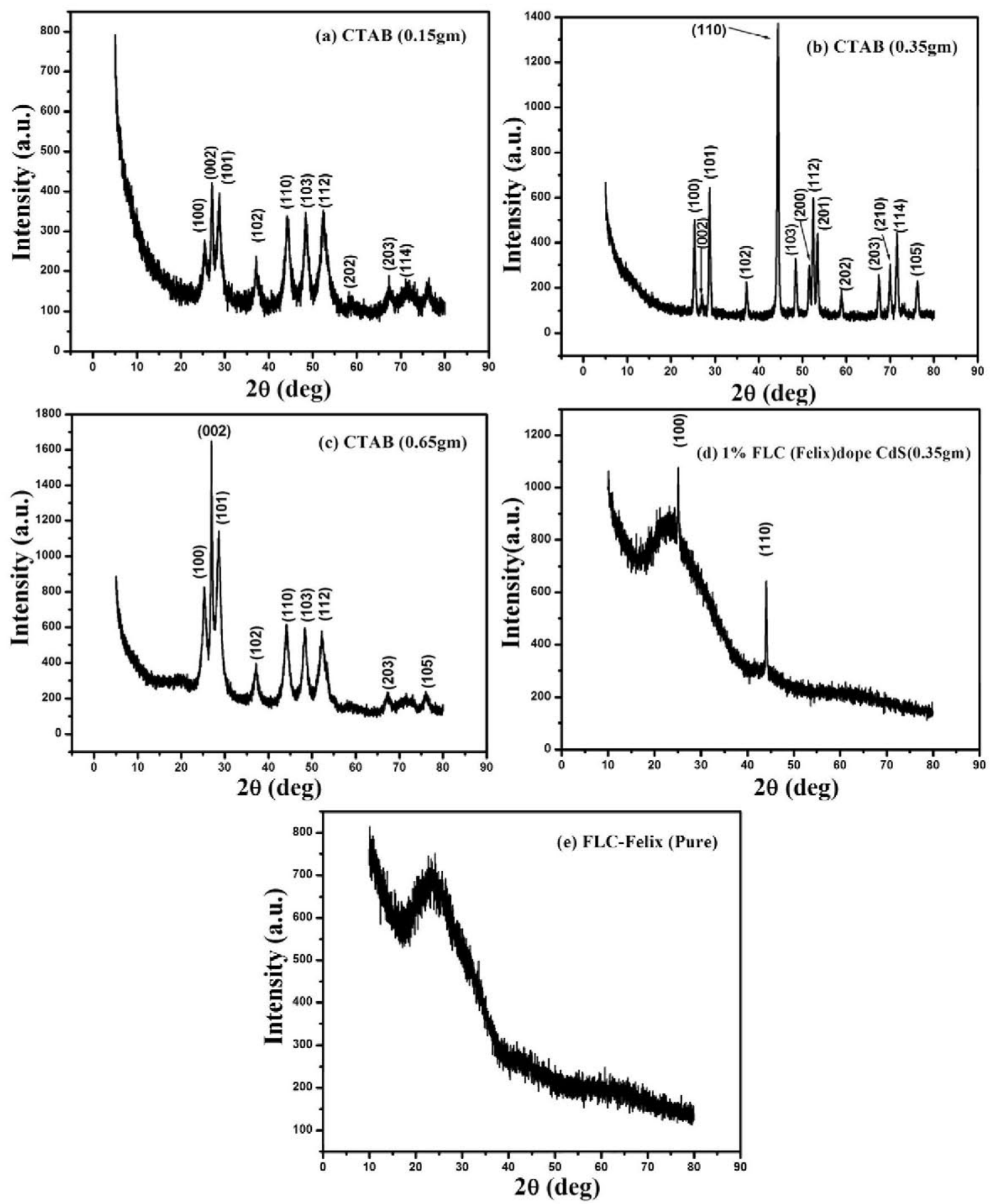

Figure 6. XRD crystalline pattern of different CdS nanostructures evolved with the addition of CTAB (a) $0.15 \mathrm{~g}$, (b) $0.35 \mathrm{~g}$, (c) $0.65 \mathrm{~g}$, (d) Felix doped CdS nanowires (CTAB $0.35 \mathrm{~g}$ ) and (e) pure FLC-Felix $(017 / 100)$.

in figures 6(a)-(e) suggest that the obtained product is well crystallized. The $d$-spacing of the CdS nanowires was calculated using the XRD analysis and compared with the standard JCPDS data (table 1). The corresponding $(h k l)$ values are given in the table.

\subsection{Optical behavior}

UV-visible spectroscopy was employed because of its simplicity, versatility, speed, accuracy and cost effectiveness. An obvious difference between certain compounds is their color. Fundamental strong absorption peak maxima appeared at $568 \mathrm{~nm}$. Going to a higher concentration of СТАB like $0.65 \mathrm{~g}$, a clear and distinct absorption band maximum occurred in the same region at $568 \mathrm{~nm}$, but there was an extra broad shoulder hump that was blue shifted in the region at $478 \mathrm{~nm}$. The absorption peak was very low when using $0.15 \mathrm{~g}$ of CTAB and a very low intense peak was located in the $491 \mathrm{~nm}$ region, which is very similar to the case using $0.35 \mathrm{~g}$ of CTAB nanogranules structured as shown in figure 7. The absorption peak position is located at $498 \mathrm{~nm}$ region with a shoulder peak which is blue shifted at $375 \mathrm{~nm}$, and results only 
Table 1. The comparison of $d$-values, obtained from XRD and JCPDS, and illustration of the corresponding $(h k l)$ planes.

\begin{tabular}{cllll}
\hline Peak & $2 \theta^{\circ}(\mathrm{deg})$ & $d$-XRD $(\AA)$ & $d$-JCPDS $(\AA)$ & $(h k l)$ \\
\hline 1 & 25.04 & 3.5561 & 3.5861 & 100 \\
2 & 26.78 & 3.3289 & 3.3599 & 002 \\
3 & 28.57 & 3.1296 & 3.1630 & 101 \\
4 & 37.07 & 2.4251 & 2.4519 & 102 \\
5 & 43.88 & 2.0632 & 2.0705 & 110 \\
6 & 48.08 & 1.8924 & 1.8998 & 103 \\
7 & 51.3 & 1.8005 & 1.8054 & 200 \\
8 & 52.09 & 1.7557 & 1.7627 & 112 \\
9 & 53.3 & 1.7042 & 1.7098 & 201 \\
10 & 69.5 & 1.5803 & 1.5896 & 203 \\
11 & 69.93 & 1.7223 & 1.7228 & 210 \\
12 & 71.3 & 1.5503 & 1.5563 & 114 \\
13 & 76.27 & 1.9903 & 1.9985 & 105 \\
\hline
\end{tabular}

due to FLC-Felix doped with uniform CdS nanowires (CTAB $0.35 \mathrm{~g}$ ). In the case of photo-excitation, this luminescence is called photoluminescence. Photo-excitation causes electrons within a material to move into permissible excited states. When these electrons return to their equilibrium states, the excess energy is released and may include the emission of light (a radiative process) or may not (a non-radiative

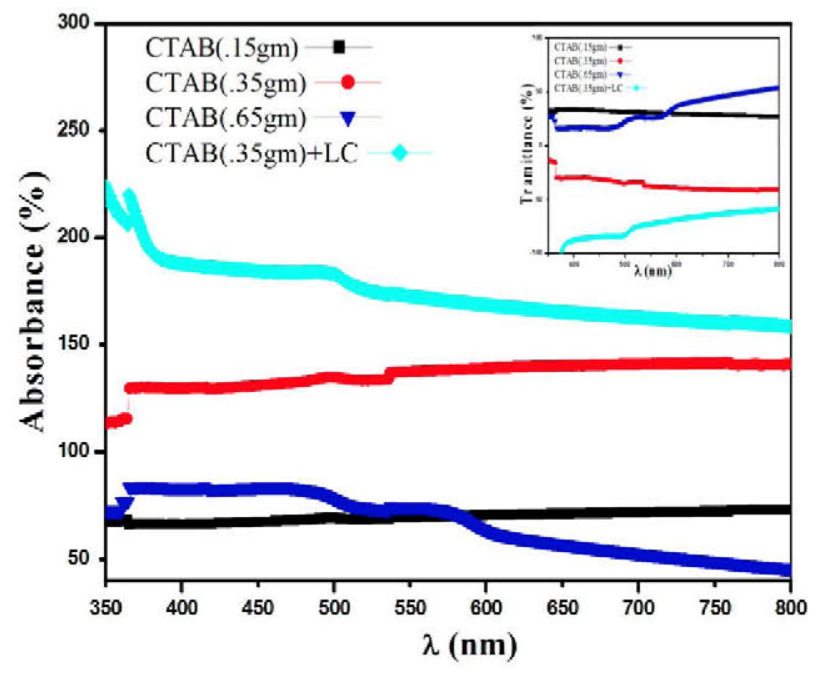

Figure 7. UV-visible absorption spectra of CdS nanostructures for various CTAB concentrations: (a) 0.15 , (b) 0.35 and (c) $0.65 \mathrm{~g}$; (d) Felix doped CdS nanowire (CTAB $0.35 \mathrm{~g}$ ) thin film coating on a glass substrate.

process). The energy of the emitted light (photoluminescence) relates to the difference in energy levels between the two electron states involved in the transition between the excited
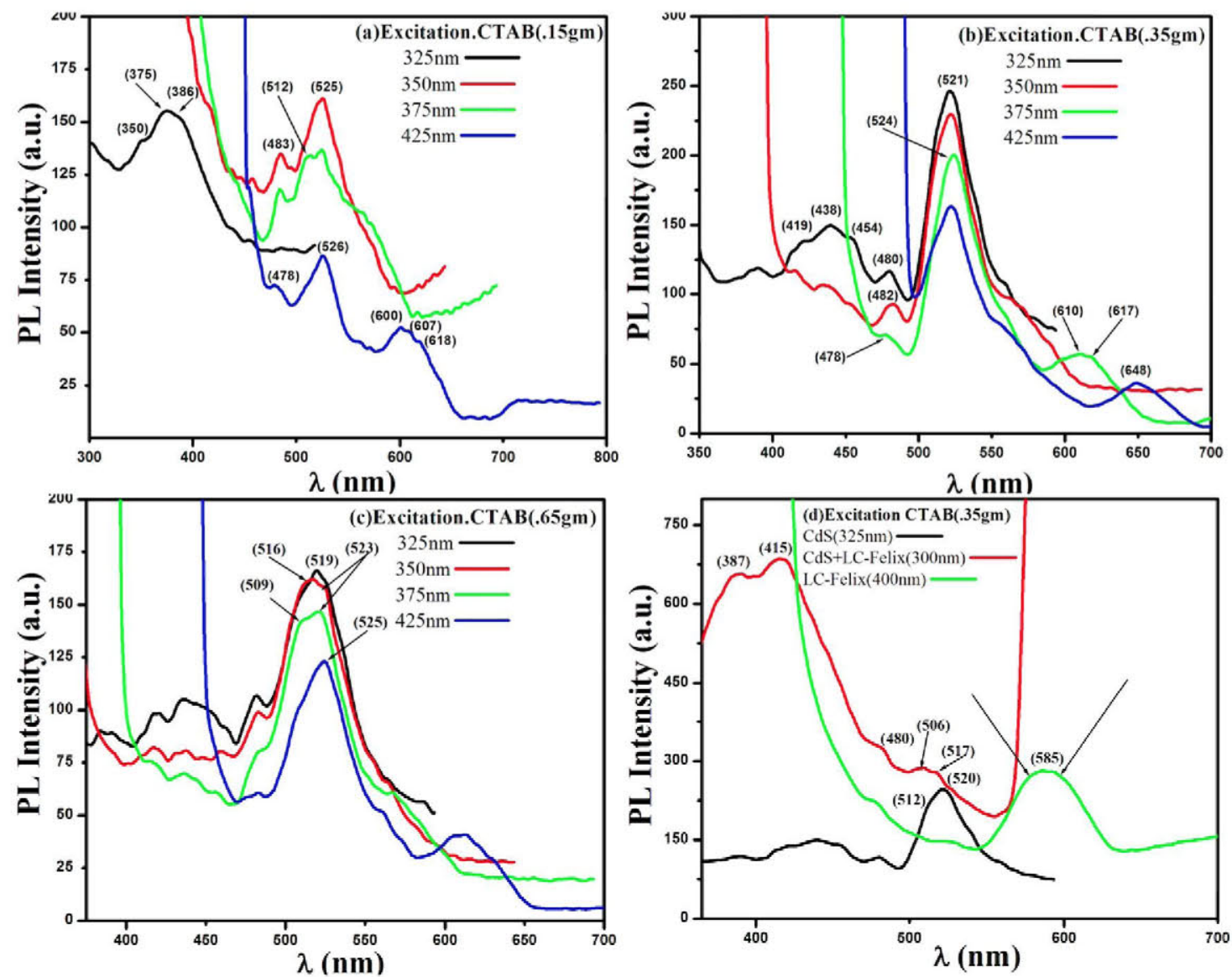

Figure 8. Photoluminescence emission spectra of CdS nanostructure evolution with CTAB concentration: (a) $0.15 \mathrm{~g}$, (b) $0.35 \mathrm{~g}$ and (c) $0.65 \mathrm{~g}$; (d) Felix doped CdS nanowire (used CTAB $0.35 \mathrm{~g}$ ) coated films at room temperature excited at the $325,350,375,400 \mathrm{and} 425 \mathrm{~nm}$ wavelength regions. 

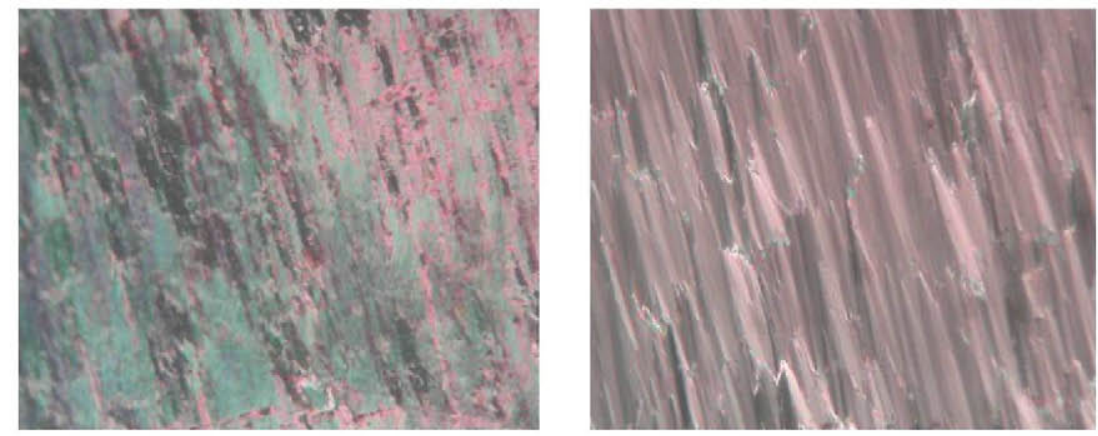

$\left(25^{\circ} \mathrm{C}\right)$

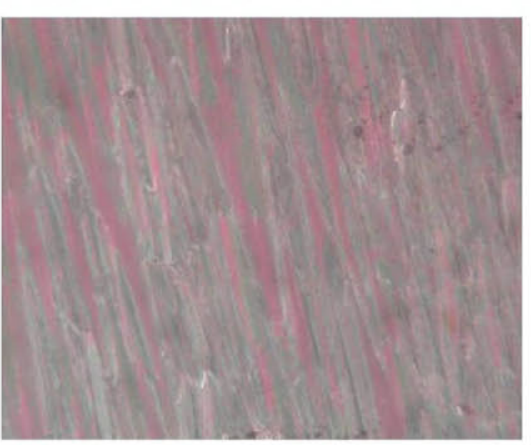

$\left(50^{\circ} \mathrm{C}\right)$

$\left(55^{\circ} \mathrm{C}\right)$

Figure 9. Optical micrograph of texture variation with temperature, for (a) $25^{\circ} \mathrm{C}$, (b) $50^{\circ} \mathrm{C}$ and (c) $55^{\circ} \mathrm{C}$, for an applied electric field on CdS nanowire (CTAB $0.35 \mathrm{~g}$ ) doped FLC-Felix sandwiched and confined in ITO polished cells.

state and the equilibrium state. Figure 8 illustrates in detail photoluminescence studies in which we use $0.35 \mathrm{~g}$ of CTAB with a high intensity peak maximum mostly occurring in the $521 \mathrm{~nm}$ region with shoulder peaks in both the red and blue shift regions, as depicted in figure 8(b). At lower concentration of CTAB $(0.15 \mathrm{~g})$, the peak intensity reduces in the wavelength region of $525 \mathrm{~nm}$, with shoulder peaks on both sides, as shown in figure 8(a). A clear distinct and sharp peak occurs in the 519 and $525 \mathrm{~nm}$ wavelength region with shoulder peak broadening in the 509 and $516 \mathrm{~nm}$ region, due to the introduction of a high concentration of $0.65 \mathrm{~g}$ of CTAB, which is noticeable in figure $8(\mathrm{c})$. For $1 \%$ of FLC doped CdS nanowires (CTAB $0.35 \mathrm{~g}$ ), the band maximum appears at $415 \mathrm{~nm}$ with a small hump in the $387 \mathrm{~nm}$ region and also many shoulder peaks appear at 480, 506 and $517 \mathrm{~nm}$ in the red shift region for an excitation range at $300 \mathrm{~nm}$. An interesting peak broadening is observed in the $585 \mathrm{~nm}$ region due to pure FLC-Felix only and a low intensity absorption peak is located at $520 \mathrm{~nm}$ with a small hump in the region of $512 \mathrm{~nm}$, which results solely due to the CdS nanowires by using $0.35 \mathrm{~g}$ of CTAB, as depicted in figure $8(\mathrm{~d})$. When the chemical substrate undergoes internal energy transitions before relaxing to its ground state by emitting photons, some of the absorbed energy is dissipated so that the emitted light photons are of lower energy than those absorbed.

\subsection{Polarizing optical microscopy (POM)}

So far, the experimental characterizations have been carried out in thin sandwich cells [40-42] where the mesogens can be aligned by surface treatment and the layers may be oriented by some shearing techniques. Electric fields can be applied by means of transparent electrodes at the glass plates. While this geometry may be important from the applications aspect, it poses some problems during preparation; in particular, the layer alignment is not easily achieved. Moreover, the evaluation of electro-optical data is quite complex because of the influence of cell boundaries. In many types of layered liquid crystalline systems, freely suspended films [43-45] have proven to be powerful instruments for the investigation of structure and dynamics. The thickness of such films can vary from thousands down to two layers. One can often treat these systems as quasi-two-dimensional, with a large experimentally accessible planar area. The orientation and dynamics of the LC director can be observed in samples with macroscopically well ordered layers. At low temperature below $50^{\circ} \mathrm{C}$, CdS nanowires are clearly dispersed in full in FLC medium. After increasing the temperature above $50^{\circ} \mathrm{C}$, the dispersion uniformly spreads. Therefore, a transition of phases may occur at $50^{\circ} \mathrm{C}$. The stripe pattern vanishes above $50^{\circ} \mathrm{C}$. Since the texture pattern changes at $50^{\circ} \mathrm{C}$, we can consider $50^{\circ} \mathrm{C}$ as a transition point (figure 9).

\subsection{Electro-optical switching response}

The phase sequences from 25 to $80^{\circ} \mathrm{C}$ clearly indicate the existence of a ferroelectric phase in the lower temperature region and a paraelectric phase in the high temperature region for pure FLC molecules. However, due to the introduction of CdS nanostructures into the FLC molecules, higher order phases appear. Therefore, the nano-confinement of CdS inside the FLC matrix increases the order parameter of the mixed system as a whole. Two peaks are anti-ferroelectric in nature and three peaks are ferroelectric in nature. Therefore, we can 


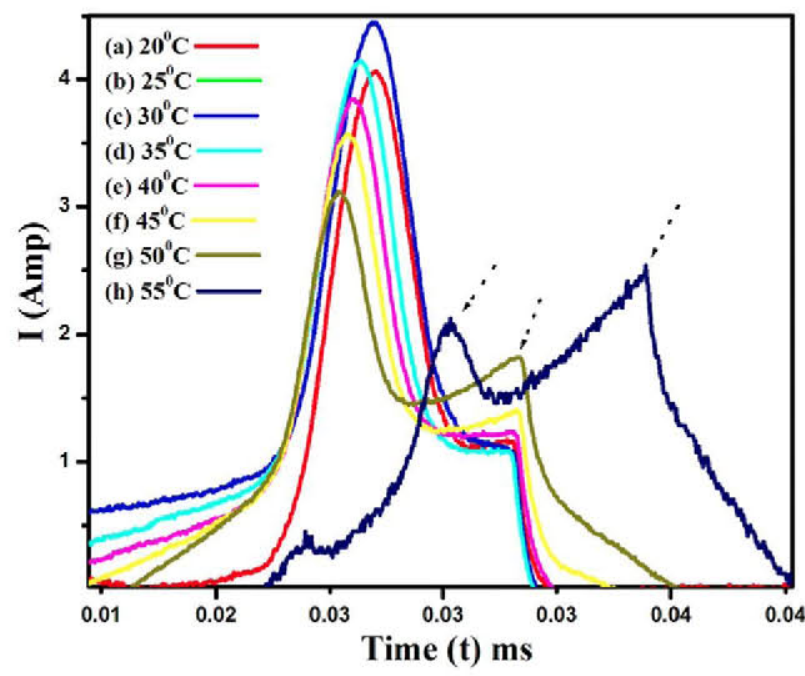

Figure 10. Electro-optical response characteristics with time $t$ (ms) with different ambient conditions of temperature variation for $\mathrm{CdS}$ nanowire (CTAB $0.35 \mathrm{~g}$ ) doped FLC-Felix (017/100).

consider $50^{\circ} \mathrm{C}$ as a transition point because the two peaks with electro-optical signature are seen to be changed at $50^{\circ} \mathrm{C}$ and converted into three peaks with electro-optical signature (figure 10); the transition point is also well recognized by temperature observation. Therefore, $50^{\circ} \mathrm{C}$ is taken as the anti-ferroelectric to ferrielectric transition point. From 25 to $50^{\circ} \mathrm{C}$, the current shows two peaks. The sharp prominent peak arises due to anti-phase oscillation and the other peak corresponds to in-phase oscillation. Therefore, this region of temperature clearly displays an anti-ferroelectric behavior. There are three peaks at $55^{\circ} \mathrm{C}$ that are ferrielectric in nature. Therefore, we obtained an anti-ferroelectric to ferrielectric transition. In addition to this, at a temperature of $20^{\circ} \mathrm{C}$, the value of spontaneous polarization, $P_{s}$, is about $70 \mathrm{nC} \mathrm{cm}^{-2}$. With increase of temperature, it decreases sharply until $55^{\circ} \mathrm{C}$ with $10 \mathrm{nC} \mathrm{cm}^{-2}$ (figure 11). Therefore, after the transition at $50^{\circ} \mathrm{C}$, the polarization shows a lower value than that before the transition point, and this is supported by earlier observations for pure AFLC [30,31]. The response time which determines the switching speed of the system is decreased with the increase of temperature, and this is also supported by earlier publications. We obtained a response time from 160 to $180 \mu$ s (figure 11), which confirms a fast switching similarly to neat FLC molecules.

The behavior of the rotational viscosity shows similar characteristics to the polarization and its value lies between 500 and $3000 \mathrm{mN} \mathrm{s} \mathrm{m}^{-2}$ (figure 12). With the increase of temperature from room temperature to the transition point (anti-ferroelectric to ferroelectric, as we confirmed from our observations), the rotational viscosity decreases due to the increase of degrees of freedom of LC mixed CdS nanomaterials. At room temperature the nanomaterial in the LC matrix is frozen because of the strong confinement (LC interactions become stronger) of the LC matrix. This confinement is decreased due to the thermal energy and the rotational mobility of the nanomaterial is increased.

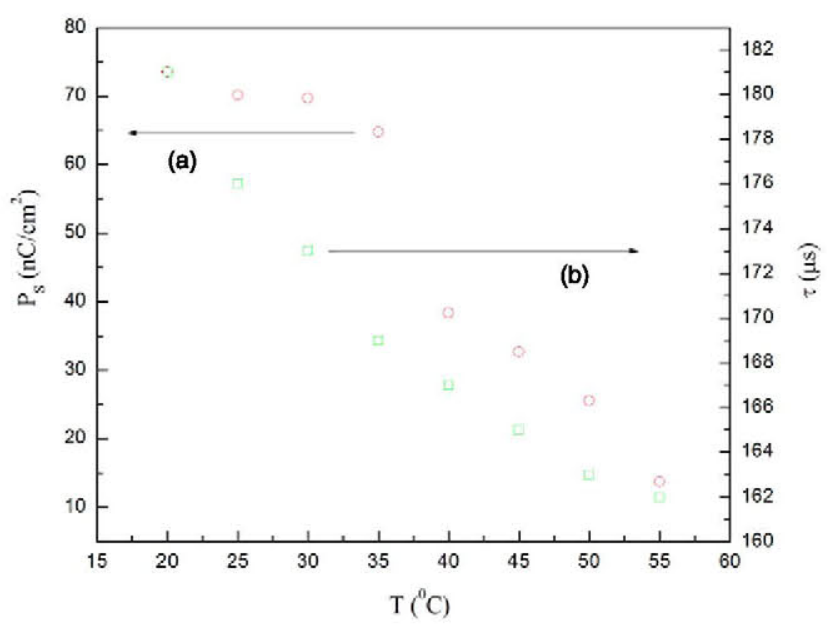

Figure 11. Temperature dependence variation with (a) spontaneous polarization $\left(P_{\mathrm{S}}\right)$ for CdS nanowire (CTAB $0.35 \mathrm{~g}$ ) doped FLC-Felix $(017 / 100),(b)$ response time $(\tau)$ for an applied electric field.

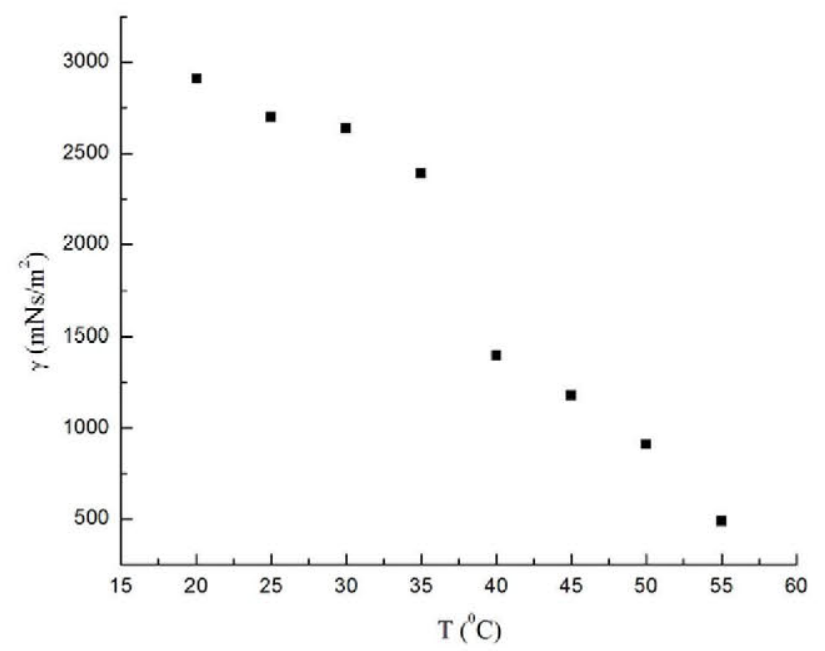

Figure 12. Temperature dependent rotational viscosity $(\gamma)$ for CdS nanowire (CTAB $0.35 \mathrm{~g})$ doped FLC-Felix (017/100) for an applied electric field.

\section{Conclusion and outlook}

In brief, we have presented a brand new and simple method for controllable growth of low dimension CdS nanowires, and a particle requiring only the possible assistance of a certain surfactant $\mathrm{CTAB}$ concentration $(0.15$ to 0.35 to $0.65 \mathrm{~g}$ ) in the precursor produces a novel fabrication. The x-ray diffraction peak pattern is remarkably shifted for good crystalline structure of the CdS nanowires and nanoparticle size. Meanwhile, the smooth specimen of LC doped CdS grains well dispersed homogeneously was confirmed by surface analysis through an AFM profilogram of height and thickness. Unlike any other currently used methods, this method has unique advantages after doping with FLC-Felix for electro-optical switching. The more sophisticated fine textured patterns of the nanomaterial doped liquid crystal enrich the quality of modern display devices and can find potential use in nanoelectronics. 


\section{Acknowledgments}

The authors wish to thank the funding agency DST, Government of India for the success of this work with the sanctioned project SR/S2/CMP-0020/2009. We are also grateful to Professor Tarak Das Basu from the Biophysics and Bio-Chemistry Department, University of Kalyani for providing us with the AFM facility. Mr Kaushik Pal is grateful to DST for providing him with a doctoral fellowship.

\section{References}

[1] Hu J T, Odom T W and Lieber C M 1999 Acc. Chem. Res. 32 435-45

[2] Li Y, Liao H, Ding Y, Qian Y, Yang L and Zhou G 1998 Chem. Mater: 10 2301-3

[3] Yu S, Wu Y, Yang J, Han Z, Xie Y, Qian Y and Liu X 1998 Chem. Mater. 10 2309-12

[4] Shen C, Zhang X and Li H 2001 Mater: Sci. Eng. A 303 19-22

[5] Yang C, Awschalom D D and Stucky G D 2002 Chem. Mater. 14 1277-84

[6] Weinhardt L 2003 Appl. Phys. Lett. 82 571-3

[7] Yang J, Zeng J, Yu S, Yang L, Zhou G and Qian Y 2000 Chem. Mater: 12 3259-63

[8] Wu J, Jiang Y, Li Q, Liu X and Qian Y 2002 J. Cryst. Growth 235 421-4

[9] Chen M, Xie Y, Lu J, Xiong Y, Zhang S, Qian Y and Liu X 2002 J. Mater. Chem. 12 748-53

[10] Li Y, Wan J and Gu Z 2000 Mater. Sci. Eng. A 286 106-9

[11] Mo X, Wang C, You M, Zhu Y, Chen Z and Hu Y 2001 Mater. Res. Bull. 36 2277-82

[12] Nikitenko S I, Koltypin Y, Mastai Y, Koltypin M and Gedanken A 2002 J. Mater. Chem. 12 1450-2

[13] Zhan J, Yang X, Wang D, Li S, Xie Y, Xia Y and Qian Y 2000 Adv. Mater. 12 1348-51

[14] Xu D, Xu Y, Chen D, Guo G, Gui L and Tang Y 2000 Chem. Phys. Lett. 325 340-4

[15] Berreman D W 1972 Phys. Rev. Lett. 28 1683-6

[16] Okulska-Bozek M, Prot T, Borycki J and Dzierski J K 1996 Liq. Cryst. $20349-59$

[17] Geary J M, Goodby J W, Kmetz A R and Patel J S 1987 J. Appl. Phys. 62 4100-8

[18] Kim Y B, Olin H, Park S Y, Choi J W, Komitov L, Matuszczyk M and Lagerwall S T 1995 Appl. Phys. Lett. $662218-9$

[19] Zhu Y M, Wang L, Lu Z H, Wei Y, Chen X X and Tang J H 1994 Appl. Phys. Lett. 65 49-51
[20] Pal K, Maiti U N, Majumder T P, Dash P, Mishra N C, Bennis $\mathrm{N}$ and Otón J M $2011 \mathrm{~J}$. Mol. Liq. $164233-8$

[21] Krueger M and Giesselmann F 2007 J. Appl. Phys. 101094102

[22] Huang J Y and Shih W T 2006 J. Phys.: Condens. Matter. $187593-603$

[23] Lynch M D and Patrick D L 2002 Nano Lett. 2 1197-201

[24] Dierking I, Scalia G, Morales P and LeClere D $2004 \mathrm{Adv}$. Mater. 16 865-9

[25] Dierking I, Scalia G and Morales P 2005 J. Appl. Phys. 97044309

[26] Lee W, Wang C-Y and Shih Y-C 2004 Appl. Phys. Lett. $85513-5$

[27] Chen H-Y and Lee W 2005 Opt. Rev. 12 223-5

[28] Lee W, Gau J-S and Chen H-Y 2005 Appl. Phys. B 81 171-5

[29] Baik I-S, Jeon S Y, Lee S H, Park K A, Jeong S H, An K H and Lee Y H 2005 Appl. Phys. Lett. 87263110

[30] Nayek P, Ghosh S, Karan S, Majumder T P and Roy S K 2008 Appl. Phys. Lett. 93112905

[31] Ghosh S, Nayek P, Roy S K, Gangopadhyay R, Molla M R and Dabrowski R 2010 Appl. Phys. Lett. 96073101

[32] Shiraishi Y, Toshima N, Maeda K, Yoshikawa H, Xu J and Kobayashi S 2002 Appl. Phys. Lett. 81 2845-7

[33] Miyama T, Thisayukta J, Shiraki H, Sakai Y, Shiraishi Y, Toshima N and Kobayashi S 2004 Japan. J. Appl. Phys. $432580-4$

[34] Pereira M S S, Canabarro A A, de Oliveira I N, Lyra M L and Mirantsev L V 2010 Eur. Phys. J. E 31 81-7

[35] Blach J-F, Saitzek S, Legrand C, Dupont L, Henninot J-F and Warenghem M 2010 J. Appl. Phys. 107074102

[36] Prakash J, Choudhary A, Mehta D S and Biradar A M 2009 Phys. Rev. E 80012701

[37] Mikulko A, Arora P, Glushchenko A, Lapanik A and Haase W 2009 Europhys. Lett. 8727009

[38] Podgornov F V, Suvorova A M, Lapanik A V and Haase W 2009 Chem. Phys. Lett. 479 206-10

[39] Clark N A and Lagerwall S T 1980 Appl. Phys. Lett. 36 899-901

[40] Kato T, Kutsuna T, Hanabusa K and Ukon M 1998 Adv. Mater. 10 606-8

[41] Pal K, Maiti U N, Majumder T P and Debnath S C 2011 J. Appl. Surf. Sci. $258163-8$

[42] Ge X, Ni Y, Liu H, Ye Q and Zhang Z 2001 Mater. Res. Bull. 36 1609-13

[43] Guo-yue X, Han W and Chuan-wei C 2006 Nonferrous Met. Soc. Chin. 16 105-9

[44] Jang J S, Joshi U A and Lee J S 2007 J. Phys. Chem. C 111 13280-7

[45] Wang Q Q, Xu G and Han G R 2005 J. Solid State Chem. $1782680-5$ 